\section{The MRL/lpr Mouse Model: An Important Animal Model for Systemic Sjögren Syndrome and Polyautoimmunity}

\section{To the Editor:}

We read with great interest the editorial by Mavragani and Moutsopoulos ${ }^{1}$, questioning the historical classification of Sjögren syndrome (SS) as primary or secondary.

Few authors ${ }^{2}$ have published papers in line with these criticisms, but this editorial was of particular interest to us, because Dr. Moutsopoulos and his team were the ones who coined the concept of secondary SS more than 35 years ago. The authors remind us that it was specifically coined for patients with SS in a rheumatoid arthritis (RA) background, because of the clinical, serological, and genetic differences they observed between SS and SS/RA patients ${ }^{1}$. The same kind of observations were made only in patients with primary biliary cirrhosis presenting with sicca syndrome ${ }^{1}$; however, the concept of secondary SS has been improperly expanded to include the other systemic autoimmune diseases, especially systemic lupus erythematosus (SLE). We think that this expansion occurred because SS, especially secondary SS, is still widely seen as a benign disease, confined to the exocrine glands, whereas it is now evident that both primary and secondary SS include patients with systemic disease, life-threatening phenotypes ${ }^{3}$, and an increased risk for lymphoma development ${ }^{1}$. Because this primary/secondary SS dichotomy misrepresents the potential manifestations and risks of the syndrome, as soon as the criteria for SS are fulfilled, we agree with the authors to say that the term secondary should be replaced by a more descriptive term (i.e., SS associated with) or polyautoimmunity, as preferred by other authors ${ }^{2}$.

Consequently, the same dichotomy has been used to classify the animal models of $\mathrm{SS}^{4}$. Choosing the right animal model for a preclinical trial in systemic autoimmune diseases (SS, SLE, systemic sclerosis...) is always challenging, because of the complex and incompletely understood pathophysiology of these diseases, but in SS it is even more complicated because of this classification. Indeed, many investigators and experts consider that all the new treatments, including immunosuppressants, biologics, and targeted therapies, should be evaluated in the primary SS mouse models [e.g., nonobese diabetic (NOD) mice and their derivatives, which are first, type 1 diabetes models] while these models are almost always limited to the glandular disease. In clinical practice, such patients would not be eligible for immunological therapies owing to the unfavorable risk-benefit balance.

We conducted a preclinical trial using an innovative anti-interferon (IFN)- $\alpha$ therapeutic vaccine in a well-recognized congenital model of SS, the MRL/MpJ-Faslpr/lpr (MRL/lpr) mouse model ${ }^{4,5}$. Despite good results (reduction of the global disease activity, type 1 IFN signature, and glandular infiltrates), we experienced difficulties in the publication process (the manuscript is still under review) because this model is usually referred to as a secondary mouse model of SS. That is because MRL/lpr mice present with glomerulonephritis, which is classic in SLE but rare (although not absent) in $\mathrm{SS}^{6}$. Of note, the MRL/lpr mouse model has also been historically identified as a pivotal model for neurological $\mathrm{SLE}^{7}$, because of the neuropsychiatric/central nervous system (CNS) manifestations (which are not absent either in SS) seen in these mice. MRL/lpr mice also present with peripheral neuropathy, a particularly rare complication of SLE, but one of the most frequent in $\mathrm{SS}^{6}$. Apart from these features, the clinical, biological, and histological phenotype of MRL/lpr mice encompasses the key features of SS: female sex predilection, decreased salivary flow rate (a feature erroneously noted as absent in all the review papers ${ }^{4}$ about animal models of SS, but which was noticed by us and other teams ${ }^{8}$ in MRL/lpr mice) and tear production, lymphocytic infiltrates in the salivary and lacrimal glands, and anti-Ro/SSA and anti-La/SSB autoantibodies production. Apart from the renal and CNS/peripheral nervous system manifestations, these mice also develop other manifestations resembling those of human patients with systemic SS, such as arthritis, pneumonitis, cryoglobulinemia, and signs of lymphoproliferation (lymphadenopathies, splenomegaly) ${ }^{7}$. Even the NOD/ShiLtJ-derived model NOD.B10Sn-H2b/J (NOD.B10), which was described by Kiripolsky, et $a l^{9}$ as an excellent tool for trials designed to evaluate extraglandular manifestations in SS, has fewer systemic features than MRL/lpr mice.

Finally, the etiopathogenesis of autoimmunity in these MRL/lpr mice encompasses pathogenic mechanisms leading to SS in humans, such as the upregulation of type 1 (Killian, et al, aforementioned unpublished work) and type $2 \mathrm{IFN}^{7}$ pathways, as well as the proliferation of double-negative (CD4-CD8-) $\mathrm{T}_{\text {cells }}{ }^{10}$, due to the lymphoproliferation (lpr) mutation-related defect in Fas-mediated apoptosis of autoreactive T cells.

No perfect model for SS exists, since no single mouse model has replicated each and every aspect of human SS to date. However, we think that the MRL/lpr mouse model should be considered as a model of choice for preclinical trials evaluating new immunological treatments in SS.

MARTIN KILLIAN ${ }^{\circ}$, MD, EA 3064, Groupe Immunité des Muqueuses et Agents Pathogènes, Université de Lyon, and Internal Medicine Department, Saint-Etienne University Hospital, Saint-Etienne; FRÉDÉRIC BATTEUX @ , MD, PharmD, PhD, Team Stress oxydant, prolifération cellulaire et inflammation, INSERM U1016 Institut Cochin, Paris; STÉPHANE PAUL, PhD, EA 3064, Groupe Immunité des Muqueuses et Agents Pathogènes, Université de Lyon, Saint-Etienne, France. Address correspondence to M. Killian, Groupe Immunité des Muqueuses et Agents Pathogènes, Faculté de Médecine Jacques Lisfranc, Pôle Santé Innovations, 10 rue de la Marandière, 42270

Saint-Priest-en-Jarez, France. E-mail: martin.killian@chu-st-etienne.fr

\section{REFERENCES}

1. Mavragani CP, Moutsopoulos HM. Primary versus secondary Sjögren syndrome: is it time to reconsider these terms? J Rheumatol 2019;46:665-6.

2. Amador-Patarroyo MJ, Arbelaez JG, Mantilla RD, Rodriguez-Rodriguez A, Cárdenas-Roldán J, Pineda-Tamayo R, et al. Sjögren's syndrome at the crossroad of polyautoimmunity. J Autoimmun 2012;39:199-205.

3. Flores-Chávez A, Kostov B, Solans R, Fraile G, Maure B, Feijoo-Massó C, et al. Severe, life-threatening phenotype of primary Sjögren's syndrome: clinical characterisation and outcomes in 1580 patients (GEAS-SS Registry). Clin Exp Rheumatol 2018;36 Suppl 112:121-9.

4. Donate A, Voigt A, Nguyen CQ. The value of animal models to study immunopathology of primary human Sjögren's syndrome symptoms. Expert Rev Clin Immunol 2014;10:469-81.

5. Hayashi Y, Haneji N, Hamano H. Cytokine gene expression and autoantibody production in Sjögren's syndrome of MRL/lpr mice. Autoimmunity 1996;23:269-77.

6. Ramos-Casals M, Brito-Zerón P, Solans R, Camps M-T, Casanovas A, Sopeña B, et al. Systemic involvement in primary Sjögren's syndrome evaluated by the EULAR-SS disease activity index: analysis of 921 Spanish patients (GEAS-SS Registry). Rheumatology 2014;53:321-31.

7. Jeltsch-David H, Muller S. Neuropsychiatric systemic lupus erythematosus and cognitive dysfunction: The MRL-lpr mouse strain as a model. Autoimmun Rev 2014;13:963-73.

8. Sato M, Kuroda S, Mansjur KQ, Khaliunaa G, Nagata K, Horiuchi $\mathrm{S}$, et al. Low-intensity pulsed ultrasound rescues insufficient salivary secretion in autoimmune sialadenitis. Arthritis Res Ther 2015; 17:278.

9. Kiripolsky J, Shen L, Liang Y, Li A, Suresh L, Lian Y, et al. Systemic manifestations of primary Sjögren's syndrome in the NOD.B10Sn-H2b/J mouse model. Clin Immunol 2017;183:225-32.

10. Alunno A, Carubbi F, Bistoni O, Caterbi S, Bartoloni E, Bigerna B, et al. CD4(-)CD8(-) T-cells in primary Sjögren's syndrome: association with the extent of glandular involvement. J Autoimmun 2014;51:38-43.

First Release September 15 2019; J Rheumatol 2020;47:1; doi:10.3899/jrheum.190820 